\title{
Neurosarcoidosis in acute inpatient rehabilitation: a case study
}

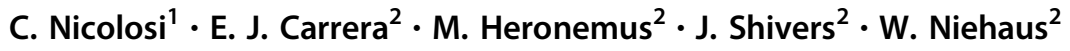

Received: 25 August 2020 / Revised: 8 October 2020 / Accepted: 17 October 2020

(c) International Spinal Cord Society 2020

\begin{abstract}
Introduction Sarcoidosis affects the nervous system in $\sim 5-10 \%$ of cases. Common presentations for neurosarcoidosis can include facial nerve neuropathy, optic neuritis, meningitis, seizure muscle weakness, and paresthesia. Due to the complex treatment of neurosarcoidosis, few reports exist involving patients' recovery in an acute rehabilitation setting.

Case presentation We describe a case of neurosarcoidosis affecting the cervical and thoracic spinal cord in an individual with known Chiari I malformation and associated syrinx decompression.

Discussion We discuss the diagnosis and treatment of neurosarcoidosis and clinical implications of acute rehabilitation on functional recovery.
\end{abstract}

\section{Introduction}

Sarcoidosis is a chronic granulomatous disease that can affect many organ systems. In $5-10 \%$ of all sarcoidosis patients, there is symptomatic involvement of the nervous system-i.e., neurosarcoidosis. Common presentations for neurosarcoidosis can include facial nerve neuropathy, optic neuritis, meningitis, seizure, and depression [1].

Diagnostic criteria for neurosarcoidosis take into account biopsy results, CSF findings, imaging, and exclusion of other diseases [1-3]. Magnetic Resonance Imaging (MRI) shows parenchymal abnormalities in more than half of neurosarcoidosis patients, most commonly non-enhancing periventricular white matter lesions [4, 5]. Combining these and other factors helps to assign the likelihood of the diagnosis as confirmed, probable, or possible according to the diagnostic criteria used.

Due to a lack of randomized placebo-controlled trials, treatment for neurosarcoidosis is based on clinical experience. Corticosteroids are often the first-line treatment, with immunosuppressants as a second line, if there is a contraindication or resistance to steroids. Even so, patients often

C. Nicolosi

Christian.Nicolosi@cuanschutz.edu

1 University of Colorado School of Medicine, Aurora, CO, USA

2 Department of Physical Medicine and Rehabilitation, University of Colorado School of Medicine, Aurora, CO, USA need rehabilitation for decreased functional capacity, weakness, dyspnea, and other disease sequelae [3].

There are only a few case reports regarding the outcomes of acute rehabilitation for patients with neurosarcoidosis. Given the highly variable presentation of neurosarcoidosis, additional examples are helpful to understand the breadth of the disease. This case documents the functional improvements of a 40-year-old African American man, who presented to acute rehabilitation after a complicated presentation not seen in previous literature.

\section{Case presentation}

Seven years prior to his presentation at our acute rehabilitation facility, at the age of 33, the patient sneezed and felt a sudden pop in the left side of his neck. Over 8 months, the patient continued to have similar episodes with sneezing or coughing. These episodes gradually increased in duration, and he also developed intermittent numbness and pain in his left upper and lower extremities. At this time, an MRI of the cervical spine demonstrated a Chiari 1 Malformation with cerebellar herniation $7 \mathrm{~mm}$ below the foramen magnum, as well as a large syrinx extending from the craniocervical junction to the upper thoracic spine. The patient had no other neurologic deficits at this time. The patient was trialed on a course of prednisone by his primary care provider. The prednisone helped but was discontinued due to concern for side effects with long-term use. The patient subsequently underwent a suboccipital craniotomy and $\mathrm{C} 1$ laminectomy in 2014 for decompression of the Chiari 1 malformation. He 
Fig. 1 Cervical MRI with and without contrast

demonstrating a large syrinx

in the cervical and upper

thoracic cord with nodular

regions of enhancement and

septations. a Axial 3D Cosmic at C7 level. b Sagittal T1 FSE

(Fast Spin Echo). c Sagittal T1

FLEX + Gadolinium. d Sagittal T2 FSE.

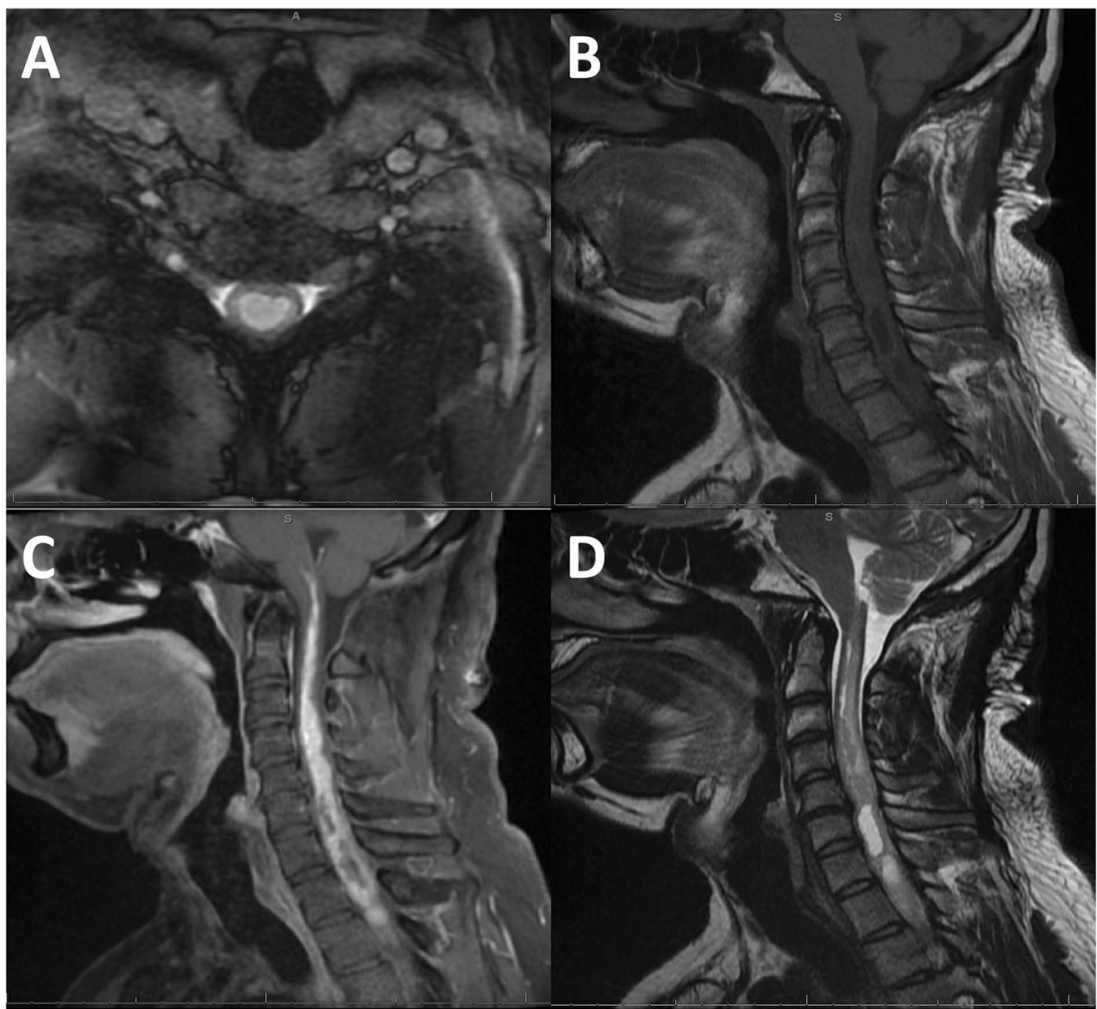

reported significant, though incomplete, improvement of prior symptoms on follow-up. Post-op imaging showed successful decompression of the foramen magnum, along with decrease in size of the syrinx. Over the next several years, the syrinx remained stable.

In early 2020, the patient began rapidly redeveloping his symptoms of left upper and left lower extremity numbness and neck pain and requested further follow up for his Chiari I malformation and syrinx. Over 2 months, he progressed to having dysphagia, ataxia, proprioceptive and balance impairments, and urinary and bowel retention. MRIs were concerning for a diffuse intramedullary neoplasm, most consistent with an ependymoma with nodular enhancement, and a syrinx extending from the left dorsal medulla to the thoracic cord which likely caused his functional decline (Fig. 1). At this time, the patient's medical history included coronary artery disease, type 2 diabetes mellitus, fatty liver disease, anxiety and depression.

Initial examination revealed $4 / 5$ distal left upper extremity and 4/5 proximal left lower extremity strength, along with decreased sensation to light touch in left upper and lower extremities. Patient had weakened active rectal tone but normal resting tone and rectal sensation. Gait exam demonstrated some instability with proper coordination of all limbs when using a walker. The patient was started on dexamethasone and underwent a C2-T3 laminectomy and an open biopsy of the intramedullary lesion. Biopsies from multiple levels revealed granulomatous inflammation of the spinal cord, concerning for neurosarcoidosis. QuantiFERON gold was negative along with an infectious workup. A Computed Tomography (CT) chest exam showed hilar and mediastinal fullness. After the biopsy results, the patient reported he had an ulcerating skin lesion biopsied 4 months prior to admission, that a dermatologist reported could also be concerning for sarcoidosis. It was decided to continue dexamethasone with an extended taper. The patient remained medically stable with mild hyponatremia. After evaluation by physical, occupational and speech therapists, as well as the Physical Medicine and Rehabilitation team, the patient was transferred to the acute rehabilitation unit.

On arrival to the rehabilitation unit, the patient was assessed using the International Standards For Neurological Standards for Neurological Classification of Spinal Cord Injury [6]. He was classified as a C1 American Spinal Injury Association (ASIA) D with right sided motor impairment in C6-T1 distribution. Our patient demonstrated patchy sensory impairments with deficits in right sided light touch sensations in dermatomes from T3-4, and S4-S5. He demonstrated left sided light touch impairments in C1, C6, T2-T5, L3, and L5-S1. The patient demonstrated deficits in right sided Pin Prick sensation from T5-T9, L1, L4-S2, and S4-S5. He demonstrated Left Pin Prick impairments from C1-C4, C7-C8, T2, and L3-S1. Specific deficits are noted in Table 1. At the time of arrival, the patient required twoperson maximum assistance for functional transfers and 
Table 1 ASIA/ISNCSCI EXAM on admission.

\begin{tabular}{|c|c|c|c|c|c|c|}
\hline & Motor right & Motor left & Light touch right & Light touch left & Pin prick right & Pin prick left \\
\hline $\mathrm{C} 1$ & - & - & 2 & 1 & 2 & 1 \\
\hline $\mathrm{C} 2$ & - & - & 2 & 2 & 2 & 1 \\
\hline $\mathrm{C} 3$ & - & - & 2 & 2 & 2 & 1 \\
\hline $\mathrm{C} 4$ & - & - & 2 & 2 & 2 & 1 \\
\hline $\mathrm{C} 5$ & 5 & 5 & 2 & 2 & 2 & 2 \\
\hline C6 & 4 & 5 & 2 & 1 & 2 & 1 \\
\hline $\mathrm{C} 7$ & 4 & 5 & 2 & 2 & 2 & 1 \\
\hline $\mathrm{C} 8$ & 4 & 5 & 2 & 2 & 2 & 2 \\
\hline $\mathrm{T} 1$ & 4 & 5 & 2 & 2 & 2 & 2 \\
\hline $\mathrm{T} 2$ & - & - & 2 & 1 & 2 & 1 \\
\hline $\mathrm{T} 3$ & - & - & 1 & 1 & 2 & 2 \\
\hline $\mathrm{T} 4$ & - & - & 1 & 1 & 2 & 2 \\
\hline T5 & - & - & 2 & 1 & 1 & 2 \\
\hline T6 & - & - & 2 & 2 & 1 & 2 \\
\hline $\mathrm{T} 7$ & - & - & 2 & 2 & 1 & 2 \\
\hline $\mathrm{T} 8$ & - & - & 2 & 2 & 1 & 2 \\
\hline T9 & - & - & 2 & 2 & 1 & 2 \\
\hline $\mathrm{T} 10$ & - & - & 2 & 2 & 2 & 2 \\
\hline $\mathrm{T} 11$ & - & - & 2 & 2 & 2 & 2 \\
\hline $\mathrm{T} 12$ & - & - & 2 & 2 & 2 & 2 \\
\hline L1 & - & - & 2 & 2 & 1 & 2 \\
\hline L2 & 5 & 5 & 2 & 2 & 2 & 2 \\
\hline L3 & 5 & 5 & 2 & 1 & 2 & 1 \\
\hline L4 & 5 & 5 & 1 & 2 & 1 & 1 \\
\hline L5 & 5 & 5 & 0 & 1 & 0 & 1 \\
\hline $\mathrm{S} 1$ & 5 & 5 & 0 & 1 & 0 & 1 \\
\hline $\mathrm{S} 2$ & - & - & 2 & 2 & 0 & 2 \\
\hline S3 & - & - & 2 & 2 & 2 & 2 \\
\hline S4-5 & - & - & 1 & 2 & 1 & 2 \\
\hline Total & 46 & 50 & 50 & 49 & 44 & 47 \\
\hline
\end{tabular}

sitting edge of bed, moderate assistance for bed mobility, minimal assistance for sit-stand transfers and contact guard assistance with grooming and hygiene. He was placed under fluid restriction, as well as prescribed sodium tabs for his hyponatremia due to syndrome of inappropriate antidiuretic hormone.

His neurosarcoidosis was managed with dexamethasone throughout his rehab admission. The patient underwent an extensive rehabilitation stay, targeted at independent self-care and ambulation with minimal assistance or supervision. The patient initially required a Foley catheter, but was able to progress to intermittent catheterization and eventually selfvoiding. During his rehabilitation stay, the patient experienced new onset right upper extremity weakness (overall 4/5 across all muscle groups) with no lab abnormalities. Given his recent surgery, after extensive discussion with neurosurgery colleagues and the patient, further imaging was not sought. While the weakness remained stable during his rehab stay, it made digital rectal stimulation, using his dominant right hand, initially more challenging.

After an 18-day rehabilitation stay, the patient was discharged home to his prior living situation with home health therapies. Upon discharge, he was ambulating using a frontwheeled walker and was completely independent in hygiene, grooming and bowel regimen. He was discharged with 11 days remaining of his dexamethasone taper. Two months after the patient's rehabilitation stay, he was ambulating independently with a cane and was performing all activities of daily living independently. Table 2 contains the patient's functional scores at several time points throughout his rehabilitation course. Quality Indicator scores in Inpatient Rehabilitation Facilities were used to assess functional improvements. The criteria for scoring are included in Table 3. 
Table 2 Rehabilitation outcomes in various periods of time in recovery.

Table 3 Quality indicator in inpatient rehabilitation facilities.

\section{Discussion}

Here we have described a patient with the acute presentation of neurosarcoidosis in the setting of prior Chiari I malformation and associated syrinx decompression. The patient
Rehabilitation day 1 Rehabilitation day 9 Rehabilitation day 18

Initial Midpoint Acute rehabilitation discharge

\begin{tabular}{|c|c|c|c|}
\hline \multicolumn{4}{|l|}{ Self-Care } \\
\hline Eating & 6 & 6 & 6 \\
\hline Grooming & 4 & 5 & 5 \\
\hline Bathing & 2 & 5 & 5 \\
\hline Dressing-Upper & 3 & 5 & 5 \\
\hline Dressing-Lower & 2 & 5 & 5 \\
\hline Toileting & 1 & 1 & 6 \\
\hline \multicolumn{4}{|l|}{ Transfers } \\
\hline Rolling left and right & 3 & 6 & 6 \\
\hline Sit to lying & 3 & 6 & 6 \\
\hline $\begin{array}{l}\text { Lying to sitting on } \\
\text { edge of bed }\end{array}$ & 3 & 6 & 6 \\
\hline Sit to Stand & 3 & 4 & 4 \\
\hline $\begin{array}{l}\text { Bed, Chair, Wheelchair } \\
\text { transfers }\end{array}$ & 3 & 4 & 6 \\
\hline Toilet & 1 & 3 & 6 \\
\hline Tub, Shower & 3 & 4 & 4 \\
\hline \multicolumn{4}{|l|}{ Locomotion } \\
\hline Walk (10 Feet) & 1 & 1 & 4 \\
\hline Walk (50 Feet with 2 turns) & NA & 1 & 1 \\
\hline Walk (150 Feet) & NA & NA & NA \\
\hline $\begin{array}{l}\text { Walk (10 feet uneven } \\
\text { surface) }\end{array}$ & NA & NA & 4 \\
\hline $\begin{array}{l}\text { Wheelchair ( } 50 \text { feet with } \\
2 \text { turns) }\end{array}$ & 4 & 5 & 6 \\
\hline Wheelchair (150 feet) & 4 & 5 & 6 \\
\hline Stairs (4 steps) & NA & NA & 4 \\
\hline
\end{tabular}

initially responded to Chiari decompression in 2014, but in 2020 redeveloped the same initial symptoms (left upper and lower extremity numbness and neck pain), along with new symptoms of bilateral lower extremity weakness, ataxia, proprioceptive impairment, dysphagia, urinary and bowel 
retention likely caused by his new thoracic lesion. While there have been other cases of neurosarcoidosis documented in rehabilitation literature, none were like this unique case.

Select neurosarcoidosis cases documented in the rehabilitation literature, have included a chronic case with acute presentation of paraplegia, limiting mobility to wheelchairlevel only due to lesion that extended from the thoracic to lumbar cord. After 3 weeks of inpatient rehabilitation and a course of prednisone, this patient was ambulatory using a walker [7]. Another case demonstrated improvement in lower extremity strength in a 66-year-old patient, who presented with numbness and paresthesia's in his bilateral lower extremities with radiating low back pain. They were found to have leptomeningeal enhancement along the distal thoracic and lumbar cord. Initially the patient had lost their ability to ambulate and was incontinent of bowel and bladder. Following a course of inpatient rehabilitation, the patient regained independent ambulation with a walker, was pain free and was continent of bowel and bladder [8].

An important distinction when comparing above cases was that our patient had co-existing Chiari I malformation with associated large syrinx. This may have anchored providers and delayed the diagnosis of neurosarcoidosis. Typically, syringomyelia involves cavities of cerebrospinal fluid and often does not require intervention [9]. Treatment often involves symptomatic management, as most syringes are not large enough for surgical correction and surgical correction can cause further morbidity. Studies have suggested an average recurrence of syringomyelia of $6.7 \%$ [10]. Of those large enough for surgical intervention, the goal of therapy is to establish normalized CSF flow [10].

Due to our patient's underlying Chiari I malformation and syrinx, it is unclear if the first manifestation of his neurosarcoidosis was in 2014, during his Chiari decompression, or 2020 in his official diagnosis. It is also unclear to what extent his symptoms responded to surgical decompression compared to the use of steroids, or both, since these were used for treatment in both hospitalizations.

This case adds to the body of evidence that patients with neurosarcoidosis can have rapid improvement in daily functioning with a comprehensive inpatient rehabilitation plan and systemic therapy. Two months after his rehabilitation stay, our patient continues to maintain his functional improvements. Further studies may look to distinguish the efficacy of rehabilitation in acute vs acute-on-chronic presentations of neurosarcoidosis, as well as the importance of establishing the diagnosis early in the disease course.

Acknowledgements We thank the multi-disciplinary staff at the UChealth Inpatient Rehabilitation unit for their dedication to outstanding patient care. This project was supported through the Department of Physical Medicine and Rehabilitation at University of Colorado School of Medicine.

\section{Compliance with ethical standards}

Conflict of interest CN, EC, MH, JS, and WN declare that they have no conflicts of interest.

Informed consent Patient consent was obtained for presentation of this case.

Publisher's note Springer Nature remains neutral with regard to jurisdictional claims in published maps and institutional affiliations.

\section{References}

1. Hebel R, Dubaniewicz-Wybieralska M, Dubaniewicz A. Overview of neurosarcoidosis: recent advances. $\mathrm{J}$ Neurol. 2015;262:258-67.

2. Drent M, Costabel U. Sarcoidosis. European Respiratory. Society. 2005. https://doi.org/10.1183/1025448x.erm3205.

3. Stern BJ, Krumholz A, Johns C, Scott P, Nissim J. Sarcoidosis and its neurological manifestations. Arch Neurol. 1985;42:909-17.

4. Joseph FG, Scolding NJ. Neurosarcoidosis: a study of 30 new cases. J Neurol Neurosurg Psychiatry. 2009;80:297-304.

5. Hoitsma EA, Drent MB, Sharma OPC. A pragmatic approach to diagnosing and treating neurosarcoidosis in the 21st century. [Miscellaneous Article]. Curr Opin Pulm Med. 2010;16:472-9.

6. Betz R, Biering-Sørensen F, Burns SP, et al. The 2019 revision of the International Standards for Neurological Classification of Spinal Cord Injury (ISNCSCI)—What's new? Spinal Cord. 2019;57:815-7.

7. Nouri KH, Kevorkian CG, Nisbet JJ. Neurosarcoidosis Presenting with Lower-Back and Leg Pain. Am J Phys Med Rehabil. 2007;86:1-2.9.

8. Hassan NF, Siazon-Leviste CL, Bennett M, Armesto DG. Progressive neurosarcoidosis: a case report. Arch Phys Med Rehabil. 1997;78:1381-3.

9. Chiari malformation and syringomyelia in: Journal of Neurosurgery: Spine Volume 31 Issue 5 (2019) [Internet]. [cited 2020 May 7]. https://thejns-org.proxy.hsl.ucdenver.edu/spine/view/journals/ j-neurosurg-spine/31/5/article-p619.xml.

10. Schuster JM, Zhang F, Norvell DC, Hermsmeyer JT. Persistent/ recurrent syringomyelia after chiari decompression-natural history and management strategies: a systematic review. Evid-Based Spine-Care J. 2013;4:116. 